DR. WALTER M STADLER (Orcid ID : 0000-0002-0435-2527)

DR. EVERETT E VOKES (Orcid ID : 0000-0002-8645-8068)

Article type :Original Article

-

(

\title{
A randomized phase II study of temsirolimus and cetuximab versus temsirolimus alone in recurrent/metastatic cetuximab-resistant head and neck cancer: The MAESTRO study
}

Running Title: The MAESTRO study

\section{Authors:}

Tanguy Y. Seiwert, MD ${ }^{1}$; Sara Kochanny, BA ${ }^{2}$; Kevin Wood, MD ${ }^{3}$; Francis P. Worden, MD ${ }^{4}$, Douglas Adkins, $\mathrm{MD}^{5}$; James L. Wade, $\mathrm{MD}^{6}$; Bethany G. Sleckman, MD${ }^{7}$; Daniel Anderson, $\mathrm{MD}^{8}$; Ryan J. Brisson, BS${ }^{9}$; Theodore Karrison, $\mathrm{PhD}^{2}$; Walter M. Stadler, MD²; Everett E. Vokes, $\mathrm{MD}^{2, *}$

\section{Affiliations:}

1. Johns Hopkins University, Sidney Kimmel Comprehensive Cancer Center, Baltimore, Maryland

2. University of Chicago Medicine and Comprehensive Cancer Center, Chicago, Illinois

3. Valley Health System, Paramus, New Jersey

4. University of Michigan, Rogel Cancer Center, Ann Arbor, Michigan

5. Washington University, Siteman Cancer Center, St. Louis, Missouri

6. Decatur Memorial Hospital, Decatur, Illinois

7. David C. Pratt Cancer Center, Mercy Hospital, St. Louis, Missouri

This is the author manuscript accepted for publication and has undergone full peer review but has not been through the copyediting, typesetting, pagination and proofreading process, which may lead to differences between this version and the Version of Record. Please cite this article as doi: $\underline{10.1002 / C N C R .32929}$

This article is protected by copyright. All rights reserved 
8. Metro-Minnesota Community Clinical Oncology Program, St. Louis Park, Minnesota

9. Oakland University, William Beaumont School of Medicine, Rochester, Michigan

\section{*Correspondence to:}

Dr. Everett E. Vokes, University of Chicago Medical Center

5841 S. Maryland Avenue, MC2115

Chicago, IL 60637, USA

Telephone: $\$ 1-773-702-9306$

E-mail: evokes@medicine.bsd.uchicago.edu

Manuscript Type: Original Article

\section{Number of pages:}

Abstract Word Count: 245

Word count: 3,636

Text pages: 15 pages

Tables: 4 tables

Figures: 2 figures

Acknowledgement of Funding Support: This study was supported by the NCI Phase 2 consortium.

\section{Conflict of Interest Statements:}

Tanguy Y. Seiwert has received research funding from Bristol-Myers Squibb, Jounce

Therapeutics, and Merck as well as honoraria from Aduro, Astra-Zeneca, Bayer, Bristol-Myers Squibb, Celgene, Cue Biopharma, Genentech, Innate, Loxo, Nanobiotix, Maverick, Merck, Oncolys, Roche Diagnostics, and SQZ Biotech.

Everett E. Vokes has a consultant/advisory role and received honoraria with AbbVie, Amgen, Astra-Zeneca, Bristol-Myers Squibb, Celgene, Eli Lilly, EMD Serono, Genentech, Merck, Novartis and Regeneron. 
Francis P. Worden has a consultant/advisory role with Merck, Cue Biopharma, Bayer, and Loxo Oncology as well as grants/research funding from Bristol-Meyers Squibb, Merck, Cue Biopharma, Eisai, Eli Lilly, Loxo Oncology and Pfizer.

Douglas Adkins has a consultant/advisory role with Pfizer, Eli Lilly, Merck, Celgene, Cue Biopharma, and Loxo Oncology as well as research funding from Phase 2 Consortium during the study, as well as grants/research funding from Pfizer, Eli Lilly, Merck, Novartis, Celgene, Astra Zeneca, Atara, Blueprint Medicine, CellCeutix, Celldex, Enzychem, Gliknik, Bristol-Meyers Squibb, Kura, Medimmune, Exelixis, Innate, Matrix Biomed, Cue Biopharma, and Sensei, outside the submitted work.

All remaining authors declare that they have no competing interests.

\section{Author Contribution Statement:}

Tanguy Y. Seiwert: Conceptualization, methodology, patient enrollment, writing - original draft, and writing - review and editing. Sara Kochanny: Data curation, visualization, writing original draft, and writing - review and editing. Kevin Wood: writing - original draft. Francis P. Worden: Patient enrollment and resources. Douglas Adkins: Investigation, patient enrollment and resources. James L. Wade: Investigation and resources. Bethany G. Sleckman: Investigation and resources. Daniel Anderson: Investigation and resources. Ryan J. Brisson: Data curation and writing - review and editing. Theodore Karrison: Formal analysis. Walter M. Stadler: Supervision and project administration. Everett E. Vokes: Conceptualization, patient enrollment, methodology, supervision, and writing - review and editing.

Acknowledgements: We thank all of the patients and their families for their participation, as well as the clinical and research staffs at the participating institutions for their hard work. We should also like to thank Shanda Lentz for her contributions while preparing this manuscript. We want to thank the following faculty for their contributions to this trial and enrolling their patients: Geraldo Colon, Sachdev P. Thomas, James A Knost, Tom Fitch, Ahmad Wehbe, David F. Sciortino, Krishna Rao, Edem Agamah, and Joseph Clark. 
Precis for use in the Table of Contents: In this randomized clinical trial of patients with cetuximab-refractory recurrent/metastatic HNSCC, cetuximab + temsirolimus induced durable responses in some patients (including $1 \mathrm{CR}$ ), indicating modest clinical activity. However, in an unselected population temsirolimus + cetuximab did not improve survival, though further investigation as a salvage option for patients with prior benefit from cetuximab monotherapy may be warranted.

Keywords: Squamous Cell Carcinoma of Head and Neck , cetuximab , temsirolimus , Neoplasm Metastasis , Neoplasm Recurrence , Local Genes , erbB-1 , TOR Serine-Threonine Kinases

\section{Abstract}

Background: Patients with cetuximab-resistant recurrent/metastatic head and neck squamous cell carcinoma (HNSCC) have poor outcomes. This study hypothesized that dual blockade of mTOR and EGFR would overcome cetuximab resistance based on the role of PI3K signaling in preclinical models of EGFR resistance.

Methods: In this multicenter randomized clinical study, patients with recurrent/metastatic HNSCC with documented progression on cetuximab (in any line in recurrent/metastatic setting) received temsirolimus $25 \mathrm{mg}$ weekly + cetuximab (TC) 400/250mg/m² weekly or single-agent temsirolimus (T). Primary outcome was progression free survival (PFS) of TC arm compared to $\mathrm{T}$ arm. Response rate, overall survival, toxicity were secondary outcomes.

Results: 80 patients were randomized to therapy with TC or T alone. There was no difference for the primary outcome of median PFS (TC arm: 3.5 months, T arm: 3.5 months). Response rate in the TC arm was $12.5 \%$ (5 responses, including $1 \mathrm{CR}(2.5 \%)$ ) compared to $2.5 \%$ in the $\mathrm{T}$ arm (1 PR) $(P=0.10)$. Responses were clinically meaningful in the TC arm (range: 3.6-9.1 months), but not in the T alone am (1.9 months). Fatigue, electrolyte abnormalities, and leukopenia were the most common grade $3+$ adverse events and occurred in less than $20 \%$ of patients in both arms.

Conclusion: The study did not meet its primary endpoint of improvement in PFS. However, temsirolimus + cetuximab induced responses in cetuximab-refractory patients with good 
tolerability. The post-hoc observation of activity in patients with acquired resistance (after prior benefit from cetuximab monotherapy) may warrant further investigation.

\section{Introduction}

$90 \%$ of head and neck squamous cell carcinomas (HNSCC) express epidermal growth factor receptor (EGFR) and its presence is associated with poor outcome[1]. Cetuximab, an IgG1 monoclonal antibody that inhibits ligand binding to EGFR and stimulates antibody-dependent cell-mediated toxicity[2], has been demonstrated to improve overall survival, progression-free survival, and response rates in the recurrent or metastatic setting when added to standard chemotherapy[3]. Single agent response rates of $9.7-13 \%$ have been noted in platinumrefractory disease with single-agent cetuximab[4], but patients eventually develop resistance and progress[5].

While resistance to cetuximab can occur through a variety of mechanisms, preclinical models suggest that downstream activation of the phosphoinositide 3-kinase (PI3K)/AKT pathway can play a key role in the development of cetuximab resistance[5]. AKT/mTOR activation is an early event in HNSCC carcinogenesis, and is implicated in progression from dysplasia to invasive carcinoma and predicts recurrence when identified at the surgical margin[6,7]. Independent activation of AKT predicts resistance to EGFR inhibitors in EGFRoverexpressing cell lines[8]. Furthermore, it has been demonstrated that genetic alterations causing PI3K/AKT/mTOR activation, such as by expression of activated PIK3CA and $R A S$ alleles, are sufficient to prevent a sustained response to cetuximab after an initial short-lasting beneficial effect[9].

Consequently, it has been hypothesized that dual blockade of EGFR and mTOR may lead to improved efficacy in tumor inhibition. Temsirolimus, an ester of the immunosuppressive drug sirolimus, acts by binding to the intracellular cytoplasmic protein, FK506 binding protein 12 (FKBP12), thereby inhibiting mammalian target of rapamycin (mTOR), a highly conserved serine-threonine kinase. In xenograft models, the combination of the EGFR small molecule tyrosine inhibitor erlotinib and temsirolimus was successful in demonstrating tumor inhibition[10]. However, a phase II trial evaluating the combination was halted early due to toxicity, notably head \& neck edema, diarrhea, and asthenia[11]. A second phase II trial evaluating the combination of everolimus, a different mTOR inhibitor and derivative of sirolimus, and erlotinib in unselected patients with platinum-resistant recurrent/metastatic 
HNSCC showed a manageable toxicity profile but did not show significant benefit[12]. Additional studies looked at combinations of mTOR inhibitors with chemotherapy or radiation[13-15].

The aim of this phase II randomized clinical trial was to evaluate progression-free survival with cetuximab + temsirolimus combination therapy compared to temsirolimus alone in patients with cetuximab-refractory metastatic or recurrent HNSCC.

\section{Materials and Methods}

Study investigations were performed following approval by the local Institutional Review Board (Univ. of Chicago IRB\#10-428-B, PI: Seiwert) and respective University of Chicago Phase 2 consortium member sites (full list is at www.clinicaltrials.gov, identifier: NCT01256385).Written informed consent was obtained from each patient.

\section{Patient Population}

Patients were $\geq 18$ years and required to have a histologic or cytologic diagnosis of HNSCC not amenable to curative intent therapy. Patients must have had progressive disease on a cetuximab-based therapy in the recurrent or metastatic setting. Acceptable prior cetuximab therapy was defined as palliative intent use either alone or in combination with chemotherapy for at least 2 weeks. Treatment with cetuximab during radiotherapy or chemotherapy was not sufficient. Patients were required to have Eastern Cooperative Group Performance Status (ECOG) 0-1, measurable disease by Response Evaluation Criteria in Solid Tumors (RECIST v1.1), normal organ and marrow function, and a life expectancy of greater than 8 weeks. Pregnant women, patients with active brain metastases, and patients with uncontrolled intercurrent illness (including, but not limited to, symptomatic congestive heart failure, unstable angina pectoris, ongoing or active infection) were ineligible for the study.

\section{Investigational Treatment}

A total of 80 patients were randomized to receive either temsirolimus + cetuximab (TC) or temsirolimus monotherapy (T). Randomization was 1:1 between the two arms using the method of permuted blocks (not blinded) and stratified based on anatomic site of tumor origin 
(oropharyngeal versus non-oropharyngeal origin). Randomization was done at the University of Chicago.

The initial cetuximab dose was a loading dose of $400 \mathrm{mg} / \mathrm{m}^{2} \mathrm{IV}$, and subsequent weekly doses were of $250 \mathrm{mg} / \mathrm{m}^{2} \mathrm{IV}$ (given over 60 minutes). In both arms, temsirolimus was given at a dose of $25 \mathrm{mg}$ weekly, infused IV over 30-60 minutes via infusion pump. Four weeks (28 days) constituted one cycle and treatment was continued until disease progression, intercurrent illness that prevented further administration of treatment, unacceptable adverse events, or the patient chose to withdraw from the study. At time of progression, patients treated on the $\mathrm{T}$ arm could cross over to the TC arm for a salvage therapy option.

Restaging radiological evaluation was performed at baseline and then every 8 weeks. Disease progression (PD) was evaluated by the investigators using RECIST v1.1. Toxicity assessments according to National Cancer Institute Common Terminology Criteria for Adverse Events version $4.0(\mathrm{NIH})$ were performed every two weeks for the first 8 weeks, and then monthly until the patient was taken off study.

\section{Statistical Methods}

The primary endpoint was progression-free survival (PFS), defined as time from randomization to disease progression or death from any cause. It was assumed that temsirolimus as a single agent would not prolong PFS based on prior studies of ineffective targeted agents in HNSCC[16]. It was hypothesized that combination treatment would increase median PFS from 2.0 months to 4.0 months, which corresponded to a hazard ratio of $\mathrm{HR}=2$. A sample size of $N=80$ patients (40 per arm) was chosen, to provide $90 \%$ power to detect such a difference based on a $\log$ rank test with a one-sided $\alpha=0.05$ (assuming 24-month accrual and 6-month follow-up periods).

Secondary endpoints were tumor response, OS, treatment related toxicity, and signal with TC combination therapy after PD on T monotherapy. PFS and OS were estimated by the KaplanMeier method and compared between treatment arms using the log rank test[17]. Confidence intervals for median survival times were derived using the method of Broomeyer and Crowley[18]. Response rates were compared by Fisher's exact test.

\section{Results}

This article is protected by copyright. All rights reserved 


\section{Patient characteristics and treatment administration}

Patients were enrolled between February 2011 and May 2013 across 14 institutions. A total of 86 patients were randomized (43 per treatment arm, see Figure 1). Three patients in each arm did not start treatment and were considered non-evaluable and per the protocol not included in the analysis. The baseline characteristics for the 80 evaluable patients are listed in Table 1. The majority of patients were male (TC: 77.5\%, T: 92.5\%), with ages ranging from 39-86 years. Approximately $40 \%$ of tumors were located in the oropharynx and $60 \%$ at non-oropharynx sites.

\section{Efficacy}

PFS and OS are depicted in Figure 2. All 80 randomized patients were evaluable for PFS and OS. PFS was not significantly different between the two arms (logrank $P=0.73$ ). Median PFS time was 105 days for the TC arm (95\% CI: 70-136 days) and 105 days for the T arm (95\% CI: 77-147 days). OS was also not significantly improved with the combination therapy (logrank $P=0.87)$. Median OS time was 177 days for the TC arm (95\% CI: $146-247$ days) and 176 days for the $\mathrm{T}$ arm (95\% CI: 131-316 days).

There was a trend toward a difference in response rates, with 5 patients (12.5\%) having either a partial $(N=4)$ or complete response $(N=1)$ in the TC arm and only 1 patient $(2.5 \%)$ having a partial response in the $\mathrm{T}$ arm $(P=0.10)$ (Table 2). The duration of responses and prior history data are shown in Table 3.

Ten patients were taken off treatment due to an adverse event (6 in TC arm and 4 in T arm), 48 due to disease progression (26 in TC arm and 22 in T arm), 2 to pursue alternative treatments ( 1 in each arm), 6 withdrew ( 1 in TC arm and 5 in T arm), 6 discontinued for other reasons ( 2 in TC arm and 4 in T arm), and 8 patients died on therapy (4 in each arm).

\section{Activity of combination therapy after failure of temsirolimus monotherapy}

In total, $15(37.5 \%)$ patients in $\mathrm{T}$ arm progressed and subsequently crossed over to receive the combination treatment. Of those 15,5 were not evaluable due to coming off treatment prior to the first evaluation. Of the remaining 10 evaluable patients, 6 had a best response of $\mathrm{PD}$ and 4 had a best response of SD.

\section{Toxicity}


In both arms, all 80 patients were evaluable for toxicity. The most common any grade and $3+$ grade AEs while patients were on their initially assigned treatment arm and deemed at least possibly related to study drug are listed in Table 4. The number of patients who experienced at least one grade $3+\mathrm{AE}$ was $28 / 40$ (70\%) in the TC arm, and 31/40 (77.5\%) in the $\mathrm{T}$ arm. In both arms, the most common at least possibly related grade 3+ AEs were leukopenia, electrolyte abnormalities, and fatigue, with no grade $3+$ AE occurring in more than $20 \%$ of patients in either arm. No grade 5 hematologic AEs were observed in either arm. Two grade 5 non-hematologic adverse events were noted in the TC arm, one with pulmonary hemorrhage and one with death not otherwise specified. A clear cause of death could not be determined (in the presence of metastatic disease, no autopsy performed). Neither grade 5 AEs were thought to be related to the study drugs. There were no grade 5 non-hematologic adverse events in the $\mathrm{T}$ arm.

\section{Discussion}

Cetuximab is an approved treatment for recurrent/metastatic HNSCC setting with modest response rates from 9.7\%-13\%, but patients eventually develop resistance[4,5]. Pre-clinical studies have demonstrated that upregulation of the PI3K/AKT/mTOR pathway is one mechanism by which cetuximab-resistance can develop[5]. Clinical studies of mTOR inhibition alone have shown poor activity in HNSCC[19]. In preclinical models blocking mTOR has been shown to reverse EGFR resistance, and has been proposed as a clinical candidate mechanism by several groups[9,10]. However prior phase II studies evaluating the tolerability and efficacy of dual inhibition of EGFR and mTOR in recurrent/metastatic, platinum-resistant HNSCC patients have either poor tolerability or, at tolerable doses, shown poor efficacy[11,12]. Poor tolerability in particular may be related to the use of small molecule EGFR inhibitor erlotinib, or continual mTOR inhibition with daily oral dosing of everolimus. Hence, in this study, we chose a different approach to accomplish EGFR and mTOR co-targeting, using the EGFR monoclonal antibody cetuximab, which, as commonly observed[20], may be easier to combine than a TKI. Additionally, we used temsirolimus given IV once weekly based on pharmacokinetic considerations with an intermittent weekly peak of mTOR inhibition[21]. Differences in tolerability between IV intermittent dosing and continual oral dosing are well described and may account for differences in tolerability and efficacy[22]. 
This phase II randomized trial of cetuximab + temsirolimus versus temsirolimus alone in cetuximab-resistant, recurrent/metastatic HNSCC patients failed to meet its primary endpoint of demonstrating a difference in PFS. However, the combination was well-tolerated and the addition of temsirolimus to cetuximab in this cetuximab-resistant population induced responses in $12.5 \%(5 / 40)$ of patients, including 1 complete response, with duration of responses ranging from 3.6 months to 9.1 months (Table 3), which may support the preclinical evidence and mechanistic rationale for dual, vertical targeting of EGFR and PIK3K/mTOR pathways $[9,10]$. The long duration of response argues against re-sensitization which would result in more transient/shorter term responses from regrowth of resistant clones. Given that the temsirolimusalone comparator arm did not show meaningful activity neither response rate nor duration of response is likely driven by temsirolimus alone. Similarly, everolimus as a single agent also did not show activity[19]. Furthermore, some patients who progressed on temsiroliums and crossed over to the combination showed some disease stabilization, indicating that efficacy is due to the combination.

Interestingly, responses occurred exclusively in non-oropharyngeal sites of origin, suggesting preferential activity in HPV-negative tumors. Activity of EGFR agents is primarily in HPV-negative tumors[23], and may relate to higher levels of EGFR expression in HPV-negative HNSCC[24].

Overall this is a negative study, and the median progression-free and overall survival do not support development in the overall population of EGFR-refractory HNSCC patients. Identification of a predictive biomarker to enrich a population with higher rate of benefit might support further development to provide a clinically meaningful treatment option for patients who fail prior cetuximab therapy. Biomarker development for cetuximab therapy in HNSCC to date has been unsuccessful, nevertheless more recent biomarker analyses with newer agents do suggest that HPV status and PTEN/PI3K influence upfront anti-EGFR therapy efficacy for HNSCC and should be explored further[23, 25].

This is a pre-immunotherapy patient cohort as is evident in the overall poor survival. However, after approval of anti-PD-1 agents in both the first and second line recurrent/metastatic setting, cetuximab and cetuximab combinations continue to play an important role in PD-L1 negative patients and patients who fail checkpoint inhibition. 
In conclusion the combination of temsirolimus and cetuximab shows modest clinical activity in cetuximab-refractory recurrent/metastatic HNSCC patients and meaningful duration of response, lending credence to the preclinical, mechanistic rationale for dual, vertical targeting of EGFR and PIK3K/mTOR pathways. However, overall this was a negative study, but further mechanistic and clinical investigation as a salvage treatment option in particular in patients with prior benefit from cetuximab monotherapy may be warranted.

\section{References:}

1. Chung CH, Ely K, McGavran L, et al. Increased epidermal growth factor receptor gene copy number is associated with poor prognosis in head and neck squamous cell carcinomas. J Clin Oncol. 2006; 24:4170-4176.

2. Kimura H, Sakai K, Arao T, et al. Antibody-dependent cellular cytotoxicity of cetuximab against tumor cells with wild-type or mutant epidermal growth factor receptor. Cancer Sci. 2007; 98:1275-1280.

3. Vermorken JB, Mesia R, Rivera F, et al: Platinum-based chemotherapy plus cetuximab in head and neck cancer. N Engl J Med. 2008; 359:1116-1127.

4. Seiwert TY, Fayette J, Cupissol, D, et al. A randomized, phase II study of afatinib versus cetuximab in metastatic or recurrent squamous cell carcinoma of the head and neck. Ann of Oncology. 2014; 25(9):1813-20.

5. Wheeler DL, Huang S, Kruser TJ, et al. Mechanisms of acquired resistance to cetuximab: role of HER (ErbB) family members. Oncogene. 2008; 27:3944-56.

6. Amornphimoltham P, Sriuranpong V, Patel V, et al. Persistent activation of the Akt pathway in head and neck squamous cell carcinoma: a potential target for UCN-01. Clin Cancer Res. 2004; 10:4029-37.

7. Nathan CO, Amirghahri N, Rice C, et al. Molecular analysis of surgical margins in head and neck squamous cell carcinoma patients. Laryngoscope. 2002; 112:2129-40.

8. Bianco R, Shin I, Ritter CA, et al. Loss of PTEN/MMAC1/TEP in EGF receptorexpressing tumor cells counteracts the antitumor action of EGFR tyrosine kinase inhibitors. Oncogene. 2003; 22:2812-22. 
9. Wang Z, Martin D, Molinolo A, et al. mTOR co-targeting in Cetuximab Resistance in Head and Neck Cancers Harboring PIK3CVA and RAS Mutations. J Natl Cancer Inst. 2014; 106 (9). doi: 10.1093/jnci/dju215.

10. Jimeno A, Kulesza P, Wheelhouse J, et al. Dual EGFR and mTOR targeting in squamous cell carcinoma models, and development of early markers of efficacy. Br J Cancer. 2007; 96:952-9.

11. Bauman J, Arias-Pulido H, Lee SJ, et al. A phase II study of temsirolimus and erlotinib in patients with recurrent and/or metastatic, platinum-refractory head and neck squamous cell carcinoma. Oral Oncol. 2013; 49(5):461-467.

12. Massarelli E, Lin H, Ginsberg LE, et al. Phase II trial of everolimus and erlotinib in patients with platinum-resistant recurrent and/or metastatic head and neck squamous cell carcinoma. Annals of Oncology. 2015; 26: 1476-80.

13. Fury M, Lee NY, Sherman E, et al. A phase 1 study of everolimus + weekly cisplatin + intensity modulated radiation therapy in head-and-neck cancer. Int J Radiat Oncol Biol Phys. 2013 Nov 1;87(3):479-86.

14. Saba NJ, Hurwitz SJ, Magliocca K et al. Phase 1 and pharmacokinetic study of everolimus in combination with cetuximab and carboplatin for recurrent/metastatic squamous cell carcinoma of the head and neck. Cancer. 2014 Dec 15;120(24):3940-51.

15. Li SH, Lin WC, Huang TL et al. Significance of mammalian target of rapamycin in patients with locally advanced stage IV head and neck squamous cell carcinoma receiving induction chemotherapy with docetaxel, cisplatin, and fluorouracil. Head Neck. 2016 Apr;38 Suppl 1:E844-52.

16. de Souza JA, Davis DW, Zhang Y, et al. A phase II study of lapatinib in recurrent/metastatic squamous cell carcinoma of the head and neck. Clin Cancer Res. 2012; 18(8):2336-2343. doi:10.1158/1078-0432.CCR-11-2825

17. Kaplan EL, Meier, P. Nonparametric estimation from incomplete observations. J Am Stat Assn. 1958; 53:457-481.

18. Brookmeyer R, Crowley J. A confidence interval for the median survival time. Biometrics. 1982; 38:29-41. 
19. Geiger JL , Bauman JE, Gibson MK, et al. Phase II trial of everolimus in patients with previously treated recurrent or metastatic head and neck squamous cell carcinoma. Head Neck. 2016 Dec;38(12):1759-1764.

20. Blaszczak W, Barczak W, Wegner A, et al. Clinical value of monoclonal antibodies and tyrosine kinase inhibitors in the treatment of head and neck squamous cell carcinoma. Med Oncol. 2017; 34(4):60. doi:10.1007/s12032-017-0918-1

21. Ekshyyan O, Mills GM, Lian T, et al. Pharmacodynamic evaluation of temsirolimus in patients with newly diagnosed advanced-stage head and neck squamous cell carcinoma. Head Neck. 2010; 32:1619-1628. doi:10.1002/hed.21374

22. Hidalgo M, Buckner JC, Erlichman C, et al. A Phase I and Pharmacokinetic Study of Temsirolimus (CCI-779) Administered Intravenously Daily for 5 Days Every 2 Weeks to Patients with Advanced Cancer. Clin Cancer Res. 2006; 12(19):5755-5763. doi: 10.1158/1078-0432.CCR-06-0118

23. Machiels JP, Haddad RI, Fayette J, et al. Afatinib versus methotrexate as second-line treatment in patients with recurrent or metastatic squamous-cell carcinoma of the head and neck progressing on or after platinum-based therapy (LUX-Head \& Neck 1): an open-label, randomised phase 3 trial. Lancet Oncol. 2015; 16(5):583-94. doi: 10.1016/S1470-2045(15)70124-5.

24. The Cancer Genome Atlas N, Lawrence MS, Sougnez C et al. Comprehensive genomic characterization of head and neck squamous cell carcinomas. Nature. 2015; 517: 576.

25. Cohen EEW, Licitra LF, Burtness B, et al. Biomarkers predict enhanced clinical outcomes with afatinib versus methotrexate in patients with second-line recurrent and / or metastatic head and neck cancer Original article. 2017;71(July):2526-2532. doi:10.1093/annonc/mdx344.

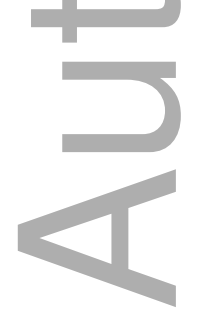

\section{Figure 1. Study Design and Patient Allocation}


Figure 2. Kaplan-Meier curves for survival. (a) Progression-free survival. (b) Overall survival. Provided $p$-values for Logrank tests. Tic marks denote censored observations.

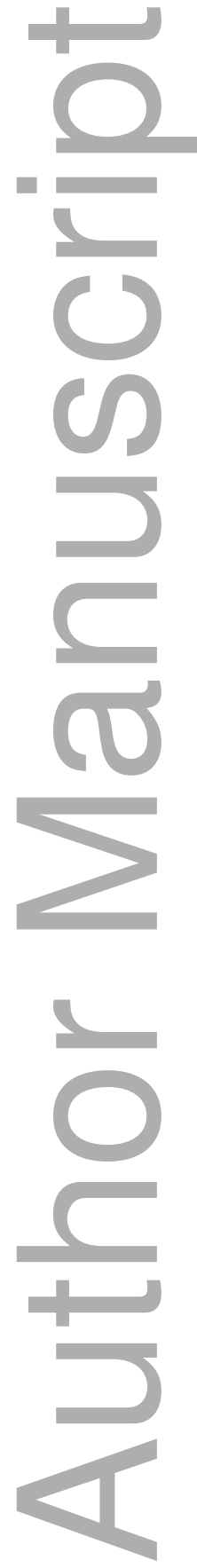

This article is protected by copyright. All rights reserved 
Table 1: Baseline de mographics and clinical characteristics.

\begin{tabular}{|l|c|c|}
\hline & \multicolumn{2}{|c|}{ No. of Patients (\%) } \\
\hline & $\begin{array}{c}\text { Temsirolimus + } \\
\text { Cetuximab } \\
(\mathrm{N}=40)\end{array}$ & $\begin{array}{c}\text { Temsirolimus } \\
(\mathrm{N}=40)\end{array}$ \\
\hline Sex & $31(77.5)$ & $37(92.5)$ \\
\hline Male & $9(22.5)$ & $3(7.5)$ \\
\hline Female & & 61 \\
\hline Age, years & $45-83$ & $36-79$ \\
\hline Median & $34(85.0)$ & $34(85.0)$ \\
\hline Range & $5(12.5)$ & $3(7.5)$ \\
\hline Race & $1(2.5)$ & $3(7.5)$ \\
\hline White & $17(42.5)$ & $16(40.0)$ \\
\hline Black & $23(57.5)$ & $24(60.0)$ \\
\hline Other & & 1.6 \\
\hline Primary Site & & \\
\hline Oropharynx & 2.7 & \\
\hline Non-oropharynx & & \\
\hline $\begin{array}{l}\text { Time between } \\
\text { cetuximab failure and } \\
\text { trial onset (months) }\end{array}$ & & \\
\hline Mean & & \\
\hline Median & & \\
\hline
\end{tabular}

Table 2: Best response rates reported in both arms

\begin{tabular}{|l|c|c|c|c|}
\hline \multirow{2}{*}{ Best response } & \multicolumn{2}{|c|}{ Temsirolimus + Cetuximab } & \multicolumn{2}{|c|}{ Temsirolimus } \\
\cline { 2 - 5 } & No. pts. & $\%$ & No. pts & $\%$ \\
\hline Complete Response & 1 & 2.5 & 0 & 0.0 \\
\hline
\end{tabular}




\begin{tabular}{|l|c|c|c|c|}
\hline Partial Response & 4 & 10.0 & 1 & 2.5 \\
\hline Stable Response & 21 & 52.5 & 20 & 50.0 \\
\hline Progressive Disease & 10 & 25.0 & 16 & 40.0 \\
\hline Death < 1st Evaluation & 3 & 7.5 & 1 & 2.5 \\
\hline $\begin{array}{l}\text { Off treatment for } \mathrm{AE}^{\mathrm{a}}<1 \mathrm{st} \\
\text { evaluation }\end{array}$ & 1 & 2.5 & 1 & 2.5 \\
\hline Not adequately assessed & 0 & 0.0 & 1 & 2.5 \\
\hline Overall Response Rate & $\mathbf{5}$ & $\mathbf{1 2 . 5 \%}$ & $\mathbf{1}$ & $\mathbf{2 . 5 \%}$ \\
\hline
\end{tabular}

${ }^{\mathrm{a}} \mathrm{AE}$, adverse event.

Table 3. Cha racteristics of responses in patients with either a $\mathbf{C R}^{\mathrm{a}}$ or $\mathbf{P} \mathbf{R}^{\mathrm{b}}$

\begin{tabular}{|c|c|c|c|c|}
\hline \multirow{2}{*}{ Response } & Site of Origin & $\begin{array}{c}\text { Duration of } \\
\text { response } \\
\text { (months) }\end{array}$ & $\begin{array}{c}\text { Duration of best } \\
\text { response to prior } \\
\text { cetuximab (best } \\
\text { response, months) }\end{array}$ & $\begin{array}{c}\text { Time between } \\
\text { cetuximab failure and } \\
\text { trial onset (months) }\end{array}$ \\
\hline Temsirolimus + Cetuximab & non-oropharyngeal & 9.1 & PR, 15.4 & 1.6 \\
\hline CR & non-oropharyngeal & 7.3 & PR, 9.0 & 1.1 \\
\hline PR & non-oropharyngeal & 5.7 & SD, 1.7 & 2.8 \\
\hline PR & non-oropharyngeal & 4.2 & PR, 9.2 & 7.8 \\
\hline PR & non-oropharyngeal & 3.6 & PR, 3.0 & 6.2 \\
\hline PR & non-oropharyngeal & 1.9 & SD, 3.7 & \\
\hline Temsirolimus
\end{tabular}

${ }^{\mathrm{a}} \mathrm{CR}$, complete response. ${ }^{\mathrm{b}} \mathrm{PR}$, partial response. ${ }^{\mathrm{c}} \mathrm{SD}$, stable disease.

Table 4. Summary of most common adverse events at least possibly related in both treatment arms $^{\text {a }}$

No. of patients (\%)

This article is protected by copyright. All rights reserved 


\begin{tabular}{|c|c|c|c|c|}
\hline \multirow{2}{*}{$\begin{array}{l}\text { Characteristics } \\
\text { Grade }\end{array}$} & \multicolumn{2}{|c|}{$\begin{array}{c}\text { Temsirolimus + } \\
\text { Cetuximab } \\
(\mathrm{N}=40)\end{array}$} & \multicolumn{2}{|c|}{$\begin{array}{c}\text { Temsirolimus } \\
(\mathrm{N}=40)\end{array}$} \\
\hline & Any & $3+$ & Any & $3+$ \\
\hline \multicolumn{5}{|l|}{ Hematologic AEs } \\
\hline Anemia & $20(50.0)$ & $1(2.5)$ & $25(62.5)$ & $3(7.5)$ \\
\hline Leukocytosis & $0(0.0)$ & $0(0.0)$ & $2(5.0)$ & $2(5.0)$ \\
\hline Lymphocyte count decreased & $10(25.0)$ & $7(17.5)$ & $16(40.0)$ & $8(20.0)$ \\
\hline Platelet count decreased & $15(37.5)$ & $1(2.5)$ & $15(37.5)$ & $0(0.0)$ \\
\hline White blood cell decreased & $9(22.5)$ & $0(0.0)$ & $11(27.5)$ & $0(0.0)$ \\
\hline \multicolumn{5}{|l|}{ Non-He matologic AEs } \\
\hline Alanine aminotransferase increased & $11(27.5)$ & $2(5.0)$ & $8(20.0)$ & $0(0.0)$ \\
\hline Alkaline phosphatase increased & $10(25.0)$ & $0(0.0)$ & $7(17.5)$ & $0(0.0)$ \\
\hline Anorexia & $9(22.5)$ & $1(2.5)$ & $15(37.5)$ & $0(0.0)$ \\
\hline Aspartate aminotransferase increased & $8(20.0)$ & $0(0.0)$ & $6(15.0)$ & $0(0.0)$ \\
\hline Cholesterol high & $7(17.5)$ & $0(0.0)$ & $12(30.0)$ & $0(0.0)$ \\
\hline Constipation & $8(20.0)$ & $0(0.0)$ & $11(27.5)$ & $0(0.0)$ \\
\hline Dry skin & $13(32.5)$ & $0(0.0)$ & $5(12.5)$ & $0(0.0)$ \\
\hline Dyspnea & $3(7.5)$ & $1(2.5)$ & $8(20.0)$ & $1(2.5)$ \\
\hline Edema face & $7(17.5)$ & $1(2.5)$ & $4(10.0)$ & $0(0.0)$ \\
\hline Fatigue & $24(60.0)$ & $3(7.5)$ & $27(67.5)$ & $3(7.5)$ \\
\hline Headache & $4(10.0)$ & $2(5.0)$ & $4(10.0)$ & $1(2.5)$ \\
\hline Hyperglycemia & $16(40.0)$ & $3(7.5)$ & $22(55.0)$ & $5(12.5)$ \\
\hline Hypertriglyceridemia & $7(17.5)$ & $0(0.0)$ & $10(25.0)$ & $2(5.0)$ \\
\hline Hypoalbuminemia & $7(17.5)$ & $0(0.0)$ & $7(17.5)$ & $0(0.0)$ \\
\hline Hypocalcemia & $8(20.0)$ & $2(5.0)$ & $8(20.0)$ & $0(0.0)$ \\
\hline Hypokalemia & $12(30.0)$ & $2(5.0)$ & $8(20.0)$ & $0(0.0)$ \\
\hline Hypomagnesemia & $22(55.0)$ & $4(10.0)$ & $4(10.0)$ & $0(0.0)$ \\
\hline Hypophosphatemia & $10(25.0)$ & $6(15.0)$ & $9(22.5)$ & $1(2.5)$ \\
\hline Lung infection & $1(2.5)$ & $1(2.5)$ & $3(7.5)$ & $2(5.0)$ \\
\hline
\end{tabular}

This article is protected by copyright. All rights reserved 


\begin{tabular}{|l|c|c|c|c|} 
Mucositis oral & $16(40.0)$ & $3(7.5)$ & $14(35.0)$ & $1(2.5)$ \\
\hline Nausea & $13(32.5)$ & $0(0.0)$ & $13(32.5)$ & $0(0.0)$ \\
\hline Rash acneiform & $19(47.5)$ & $4(10.0)$ & $8(20.0)$ & $1(2.5)$ \\
\hline Rash maculo-papular & $5(12.5)$ & $0(0.0)$ & $7(17.5)$ & $0(0.0)$ \\
\hline Vomiting & $6(15.0)$ & $0(0.0)$ & $5(12.5)$ & $0(0.0)$ \\
\hline Weight loss & $6(15.0)$ & $0(0.0)$ & $8(20.0)$ & $0(0.0)$ \\
\hline
\end{tabular}

${ }^{\mathrm{a}}$ Frequency cutoff for grade 1-2 AEs is $15 \%$, and grade $3+$ AEs is $5 \%$ for either arm. Maximum grade per patient reported.

${ }^{\mathrm{b}} \mathrm{AE}$, adverse event.

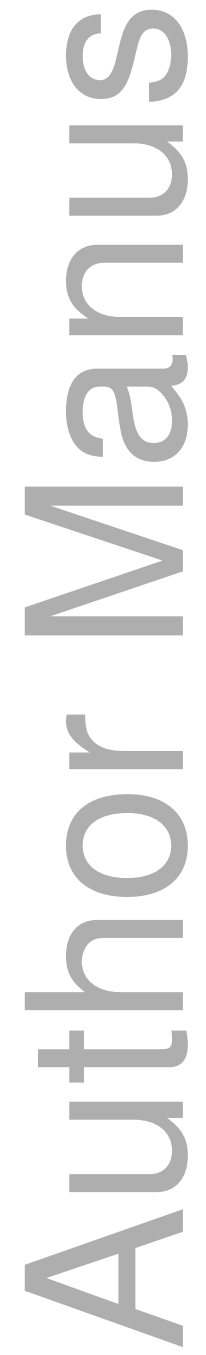


HNSCC:

1) Recurrent/

Metastatic

2) Failed prior EGFR treatment for R/M disease

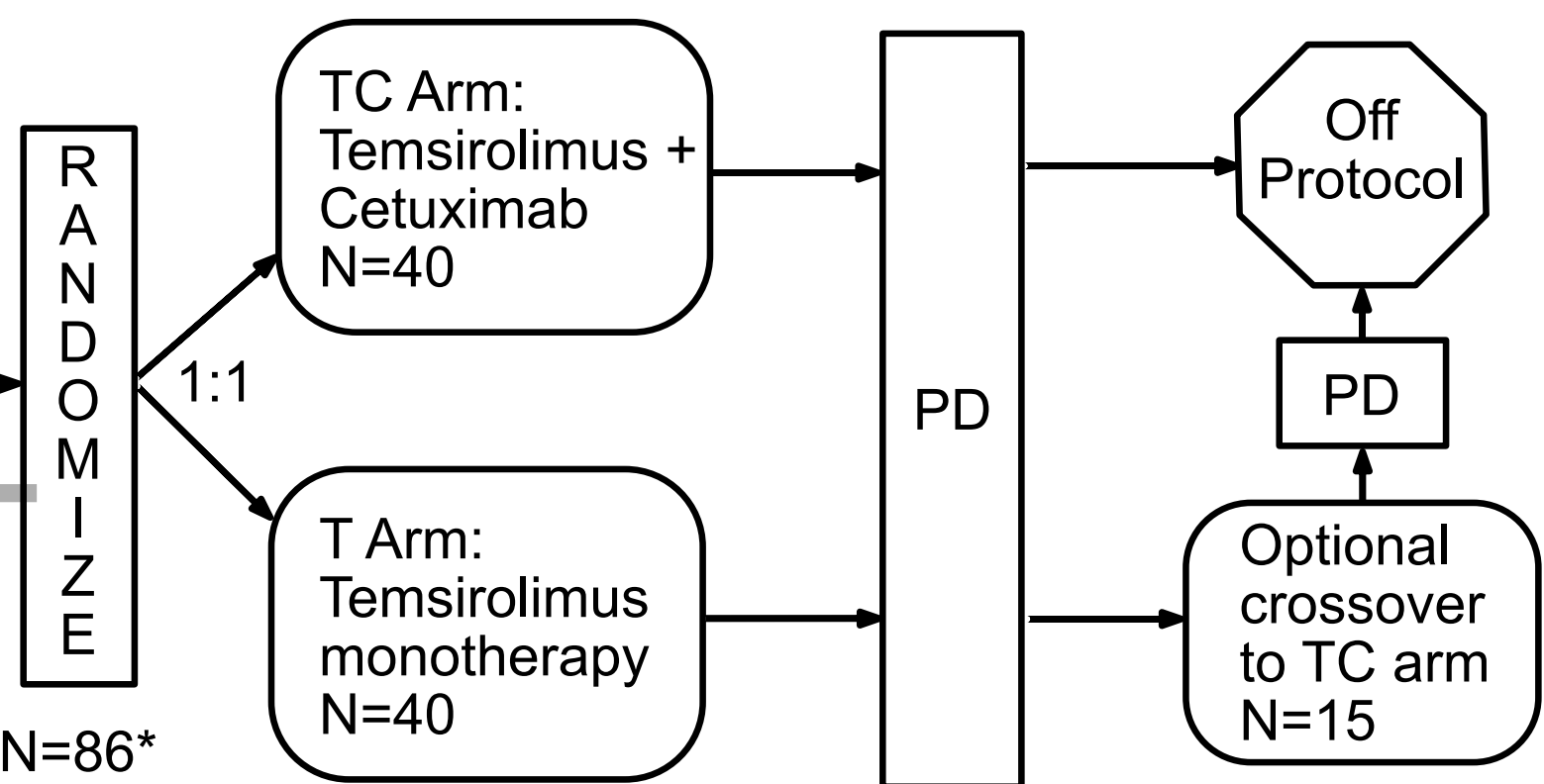

*Three patients on each arm did not start treatment

cncr_32929_f1.eps 
(a)

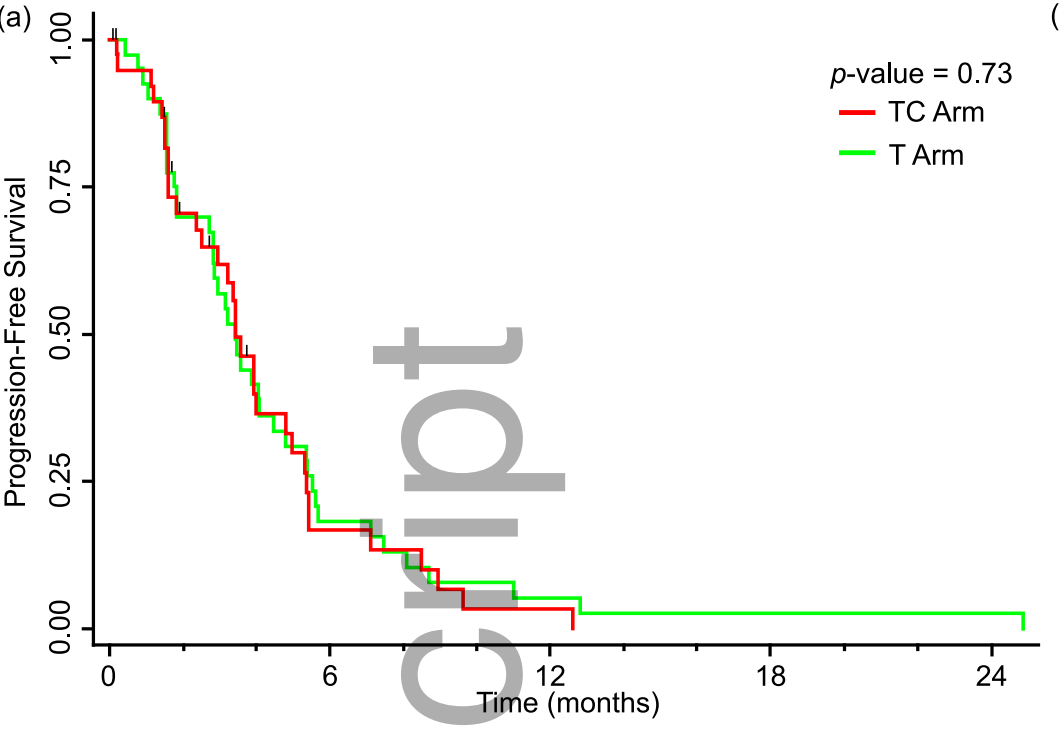

(b)

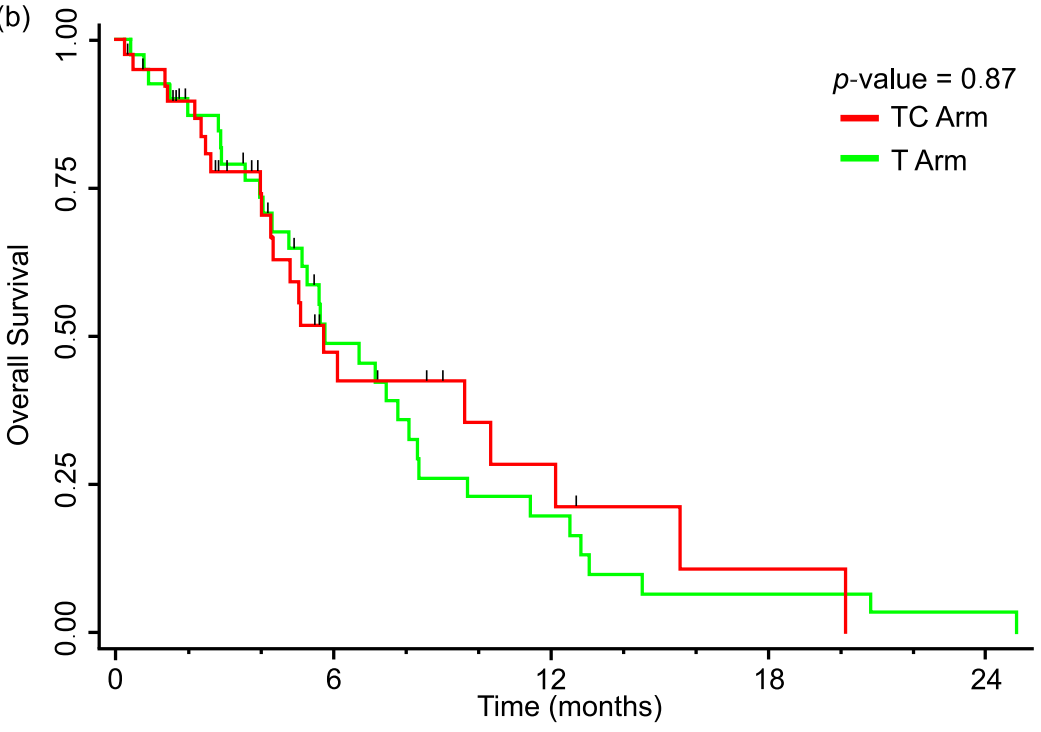

cncr_32929_f2.eps

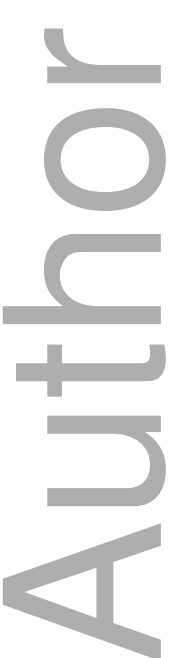

